\title{
Image Fusion and Electromagnetic Needle Tracking for the Biopsy of Pelvic Lesions - Report of 2 Cases
}

\section{Introduction \\ $\nabla$}

Percutaneous or transvaginal biopsy is often performed under ultrasound (US) guidance, which has the advantage of real-time images and no ionizing radiation. However, US may be challenging in obese patients, and areas covered by air may be difficult to visualize, for instance in the pelvis. These challenges may be overcome by using image fusion and electromagnetic needle tracking.

Image fusion involving US is a technique, in which a previously recorded CT or MRI examination is co-registered with realtime US images. It works by means of software in the system and a magnetic positioning system - a magnet placed beside the patient and magnetic sensors attached to or incorporated in the transducer - and is available from most commercial high-end systems. The images are aligned by co-registration of corresponding points visible on US and CT (or MRI), for instance anatomical landmarks as the umbilicus and the aortic bifurcation. The time spent on the co-registration decreases with experience and is approximately $5 \mathrm{~min}$. Accuracy depends on the landmarks, positioning of the patient and respiration, but has been shown to be as low as a few millimeters in patients (Hakime A et al. Cardiovasc Intervent Radiol. 2011; 34: 338-344). When the images are co-registered, the CT or MRI images are reformatted in a projection to fit the real-time US images (Ewertsen C et al. Am J Roentgenol. 2013; 200: W249-255).

Electromagnetic needle tracking is also based on a magnetic positioning system and can be used separately or together with image fusion. An electromagnetic sensor is embedded in the tip of the needle. The tip of the needle and the puncture line are marked electronically and simultaneously on the US and CT (or MRI) images, instead of the usual mechanical registration of the echoes from the needle tip (Venkatesan AM et al. Radiology. 2011; 260: 848-856). These new techniques en-

\section{License terms}

()(1) $\Theta \circledast$ able percutaneous US-guided intervention in areas with a poor US overview. In patients with a previous history of cancer or with suspicion of actual cancer disease, biopsy is crucial in order to establish a correct diagnosis and treatment plan.

We present 2 cases in which image fusion and electromagnetic needle tracking have been used to locate and biopsy poorly defined lymph nodes in the pelvis.

\section{Case Description \\ $\nabla$}

Case 1

A 64-year-old woman with a history of previous cervical cancer presented with pain and swelling of the left leg. CT showed a tumor measuring $5 \times 3.5 \mathrm{~cm}$ on the left side of the pelvis. Transvaginal and transabdominal US-guided biopsy were attempted, but without obtaining sufficient material for histology. PET/CT confirmed the suspicion of malignancy. A percutaneous core needle biopsy was obtained with the assistance of image fusion and electromagnetic needle tracking ( $\bullet$ Fig. 1). Histology revealed low differentiated carcinoma, possibly relapse of the cervical cancer. No complications of the biopsy were observed.

\section{Case 2}

A 69-year-old woman with a history of previous endometrial adenocarcinoma presented with severe swelling of the left leg. 5 years earlier, the patient had undergone hysterectomy and radiation therapy. MRI and PET/CT revealed a large lymph node conglomerate measuring $5 \times 5 \times$ $12 \mathrm{~cm}$ enclosing the left-sided iliac vessels causing a thrombus in the left external iliac vein and obstructing the left ureter. Percutaneous US-guided core needle biopsy assisted by image fusion and electromagnetic needle tracking was performed. Initially the lymph nodes were invisible, but after co-registration with the CT images they became visible ( $\bullet$ Fig. 2). Histology revealed metastasis from adenocarcinoma. No complications of the biopsy were observed.

\section{Discussion}

$\nabla$

This is the first case report describing image fusion and electromagnetic needle tracking for the biopsy of lymph nodes in the pelvis in patients with a previous history of gynecological cancer. In both cases successful biopsies with sufficient material were obtained. Biopsy from the lymph nodes was crucial in order to establish whether the patients had relapse of a previous cancer or had a new diagnosis.

The overview was poor due to overlying air in the bowel and obesity, and the close relation to the iliac vessels made correct visualization of the needle placement very important to avoid bleeding. None of the patients experienced complications at the 4-h follow-up with regular measurements of blood pressure. Both patients were subsequently discharged from the hospital and no complications were described in their records at follow-up.

Biopsy from enlarged intraabdominal lymph nodes is often obtained with image guidance from US. However, at large depths the echoes from the needle tip may be difficult to visualize. Electromagnetic needle tracking enables visualization of the needle tip during the entire procedure, because it is electronically marked on the screen. The rate of success of the method has been shown to equal conventional image-guided biopsy in a phantom (Ewertsen $\mathrm{C}$ et al. Ultraschall Med. 2011; 32: 614-618). It has also been evaluated for liver biopsy and compared to free-hand liver biopsy. The use of electromagnetic needle tracking reduced the number of redirections of the needle and also the time spent on the procedure regardless of the operator experience (Hakime A et al. J Vasc Interv Radiol. 2013; 24: 1682-1689). Realtime image fusion involving US has mainly been evaluated for transabdominal and transrectal US (Mauri G et al. Cardiovasc Intervent Radiol. 2015; 38: 143151), (Pinto PA et al. J Urol. 2011; 186: 1281-1285). The method has also been used for gynecological evaluation of deep infiltrating endometrioses (Millischer AE et al. Ultrasound obstet gynecol. 2015; 46: 109-117). When using image fusion, the examination time is slightly prolonged due to the co-registration procedure, but intervention in areas previously inaccessible to US is enabled. An alternative to US would have been laparoscopic, surgical or endoscopic biopsy 

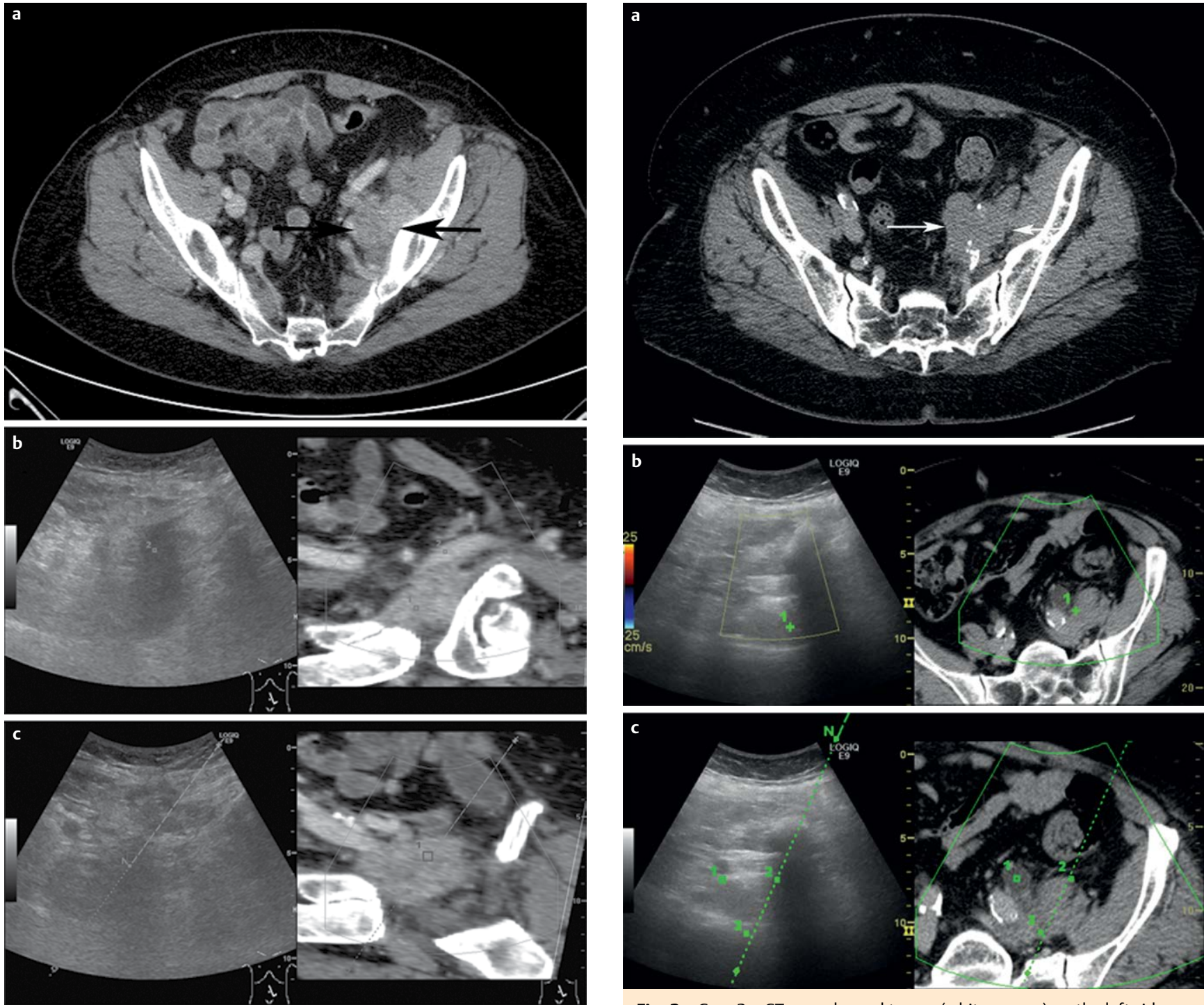

Fig. 1 Case 1 a CT scan showed tumor (black arrows) on the left side of the pelvis. $\mathbf{b}$ Image fusion, ultrasound to the left and corresponding CT plane to the right. The tumor is marked by $1 \square$ and the iliac artery is marked by $2 \square$. c Percutaneous core needle biopsy using image fusion and electromagnetic needle tracking. The gray dotted line is the virtual route of puncture, and the needle tip is marked with $\mathrm{N}+$. The tumor is marked with $1 \square$.

Fig. 2 Case 2 a CT scan showed tumor (white arrows) on the left side of the pelvis. $\mathbf{b}$ Image fusion, ultrasound to the left and corresponding CT plane to the right. The tumor is marked by $1 \square$. c Percutaneous core needle biopsy using image fusion and electromagnetic needle tracking. The green dotted line is the virtual route of puncture, and the needle tip is marked by $\mathrm{N}+$. Hydro ureter $(1+)$, tumor area $(2+, 3+)$.

(Mohamadnejad M et al. Gastrointest Endosc. 2012; 75: 146-151), which may be more traumatic for the patient. Due to the extra time spent and the cost of the needle, we recommend that the method should be reserved for difficult cases especially in anatomical areas with limited US overview.

\section{Catchwords Phrases}

$\nabla$

Image fusion and electromagnetic needle tracking help the visualization of pelvic lesions. Make a new line Electromagnetic needle tracking enables biopsy in anatomical areas with a poor overview.

D. Dencker, J. F. Topsøe, C. Ewertsen,

S. Karstrup ${ }^{\dagger}$

dittedencker@gmail.com 